\title{
A ready-to-use online tool to calculate the BCF of active substances: MOSAIC $_{\text {bioacc }}$
}

Aude Ratier ${ }^{1}$, Christelle Lopes ${ }^{1}$, Gauthier Multari ${ }^{1}$, Marc Babut ${ }^{2}$, Vanessa Mazerolles ${ }^{3}$, Sandrine Charles ${ }^{1}$

1. Univ Lyon, Université Lyon 1, CNRS, LBBE UMR5558, F-69622 Villeurbanne, France.
2. INRAE, UR RIVERLY, centre de Lyon-Villeurbanne, 5 Avenue de la Doua, CS20244, 69625 Villeurbanne Cedex, France.

3. Anses, 14 rue Pierre et Marie Curie, 94701 Maisons-Alfort Cedex, France.

\section{INTRODUCTION}

Toxicokinetic/toxicodynamic (TKTD) models are used to describe and predict the toxicity and the effects of chemical substances on individual traits based on experimental data. The toxicokinetic (TK) part describes the relationship between the exposure medium concentration and the internal concentration within an organism, considering various processes such as ADME (accumulation depuration, metabolization and excretion) ${ }^{[1]}$

Based on regulation No 283/2013 (EU) ${ }^{[2]}$ laying down the data requirements for active substances for the placing of plant protection products on the market, a bioaccumulation study on fish is required following the OECD guideline $305{ }^{[3]}$. This study relies on the fit of a TK model to bioaccumulation data from which the bioconcentration factor (BCF) can be calculated. Our too MOSAIC bitioc makes this calculation user-friendly through a web interface.

\section{OBJECTIVES}

- To develop a ready-to-use on-line tool for easily estimating BCF in a regulatory framework, in agreement with EFSA's scientific opinion on good modeling practices ${ }^{[4,5]}$;

- To include this tool as a module of the MOSAIC platform ${ }^{[6]}$, in order to contribute to the development and widespread use of TKTD models in general.

All calculations are performed under a Bayesian framework. The TK model on which they are based has recently be revisited and validated [7]. Our tool provides an easy way to estimate its parameters leading to the final calculation of the BCF as required in a regulatory context.

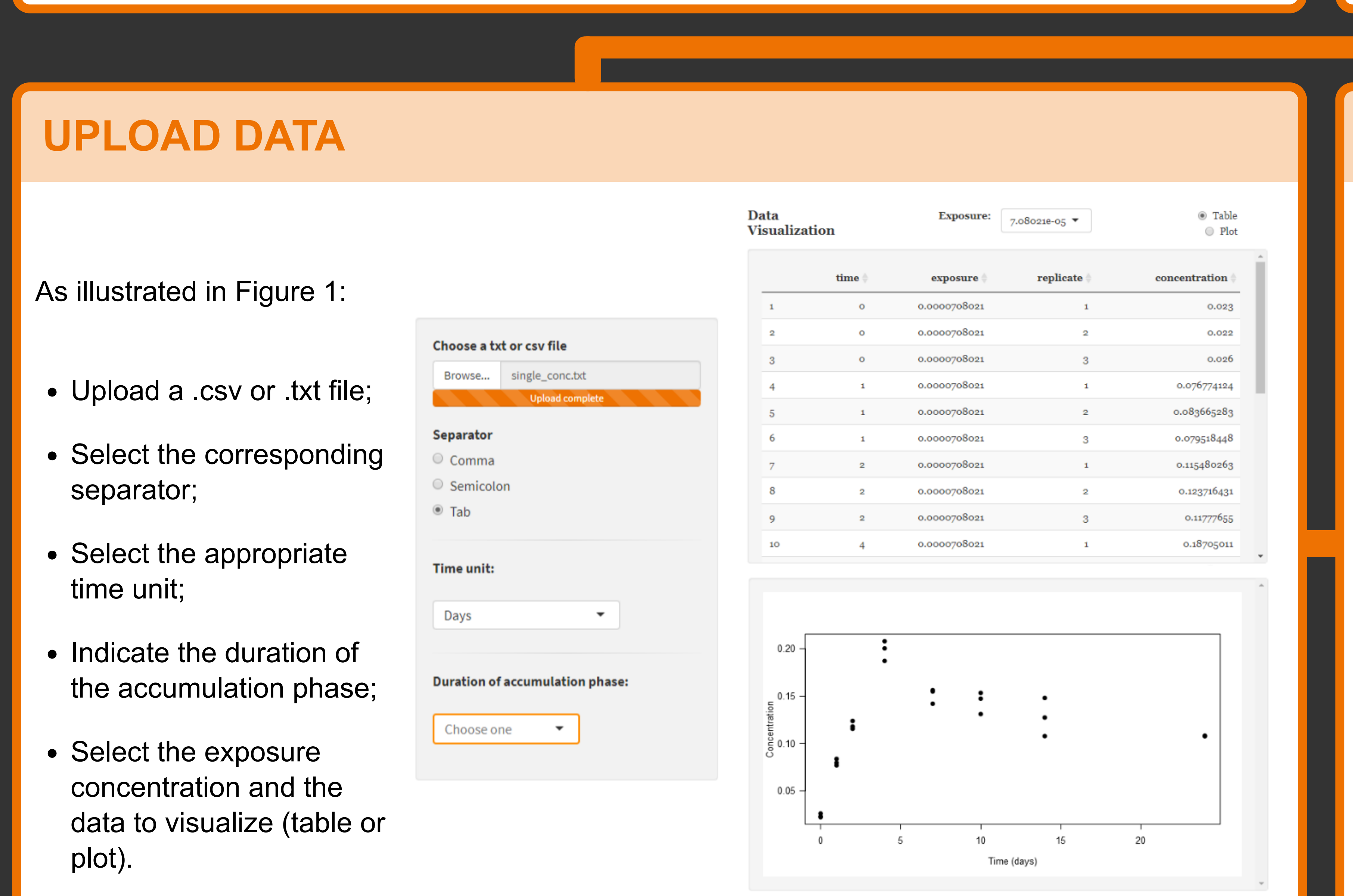

Figure 1. Data upload and visualization.

\section{MODEL AND PARAMETERS}

If organisms are considered as single compartments, a generic first-order kinetic bioaccumulation model can be expressed as follows (Eqs. (1) and (2))

$$
\left\{\begin{array}{lrl}
\frac{d C(t)}{d t}=\sum_{i=1}^{n}\left(k_{i} \times C_{i}\right)-\sum_{d=1}^{q}\left(k_{d} \times C(t)\right) & \text { for } 0<t<t_{c} \\
\frac{d C(t)}{d t}=-\sum_{d=1}^{q}\left(k_{d} \times C(t)\right) & \text { for } t>t_{c}
\end{array}\right.
$$

ogether with a Gaussian distribution for the stochastic part: $C_{o b s}(t) \sim \mathcal{N}\left(C(t), \sigma^{2}\right)$.

$(t)$ : predicted substance concentration at time $t$ within whole

organisms $\left(\mu g . g^{-1}\right.$ wet weight (ww))

$k_{i}:$ uptake rate from exposure source $i$ : water, food or sedime

$C_{i}$ : mean substance concentration in exposure source $i$ : water

$p:$ number of elimination processes

: rate related to elimination processes $d:$

$t_{c}$ : duration of the exposure phase (time)

: standard deviation of the contamin

in organisms $\left(\mu g . g^{-1} \mathrm{ww}\right)$

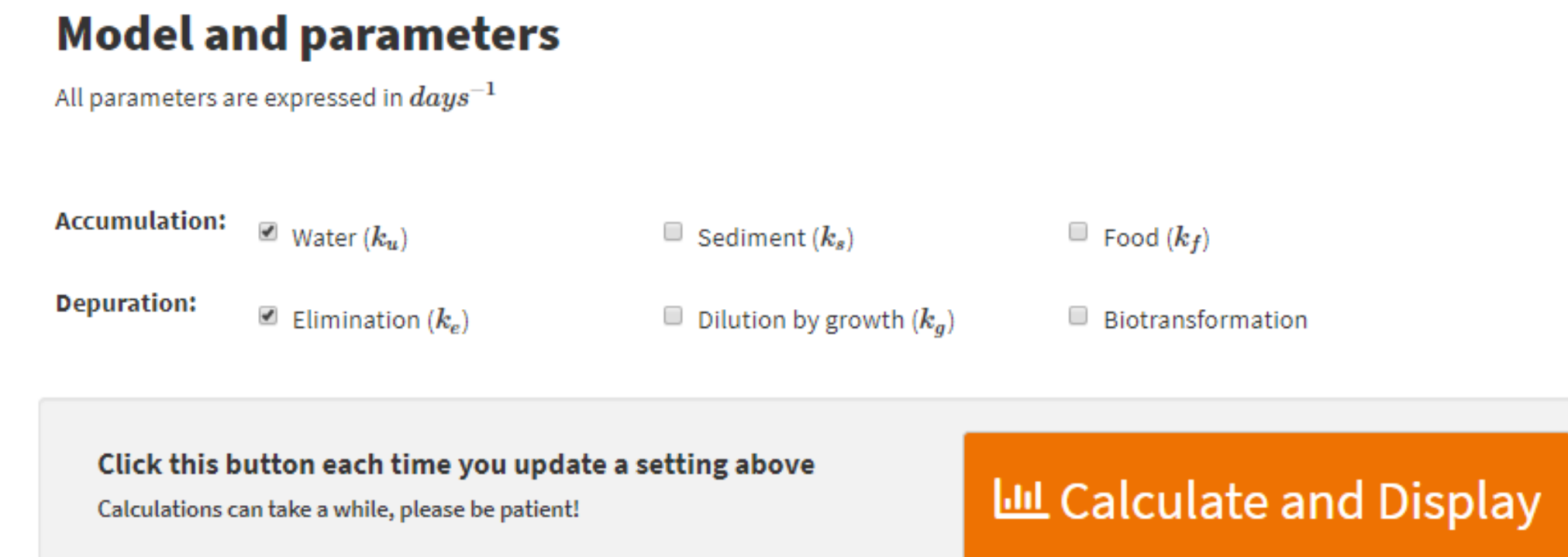

Figure 2. Model choice and run

We implemented the model under a Bayesian inference framework with R and JAGS softwares (Fig. 2).

\section{(20)}

BIOACCUMULATION FACTOR CALCULATION

- As a first result, we provide the kinetic bioconcentration factor $\left(\mathrm{BCF}_{\mathrm{k}}\right)$ and the $\mathrm{BCF}$ at steady state $\left(\mathrm{BCF}_{\mathrm{SS}}\right)$

- $B C F_{k}=\frac{k_{u}}{k_{e}}$ and $B C F_{s s}=\frac{C\left(t_{c}\right)}{C_{i}}$

- BCF are given as probability distributions (Fig. 3) and summarized with their mean and their $95 \%$ uncertainty limits $(95 \%$ credible intervals, Table 1).

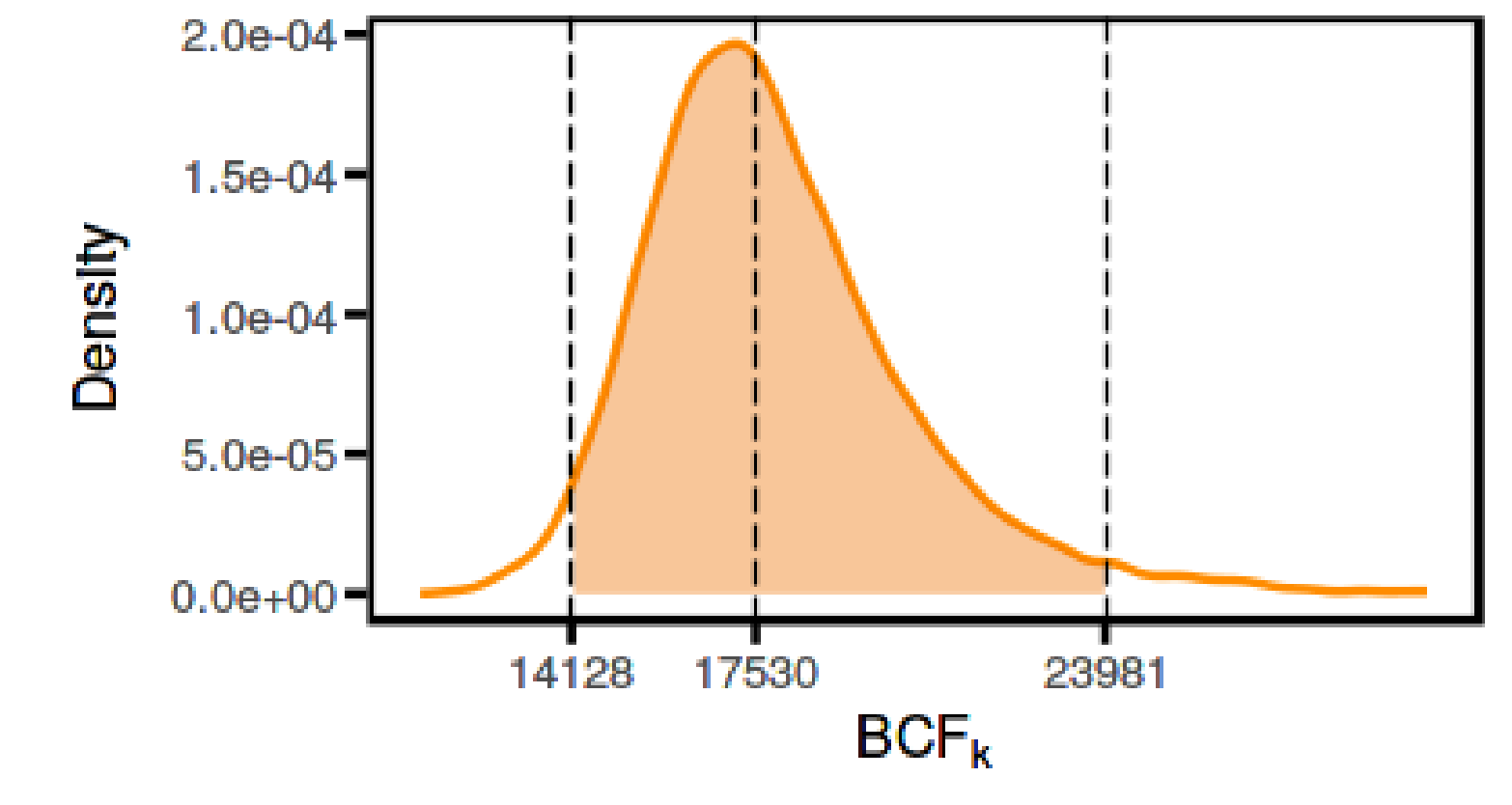

Figure 3. Example of a $\mathrm{BCF}_{\mathrm{k}}$ distribution.

Table 1. $\mathrm{BCF}_{\mathrm{k}}$ results.

\begin{tabular}{cccc} 
Quantiles & $2.5 \%$ & $50 \%$ & $97.5 \%$ \\
\hline$B C F_{k}$ & 14128 & 17530 & 23981
\end{tabular}

\section{FITTING RESULTS}

We first provide the fitted curve superimposed to the observations (Fig. 4 black dots): the orange plain line is the median curve the gray zone the uncertainty band delimited by 25 and $97.5 \%$ quantiles in orange dotted lines.

- Then we provide a summary of model paramerers as medians and $95 \%$ uncertainty limits (Table 2).

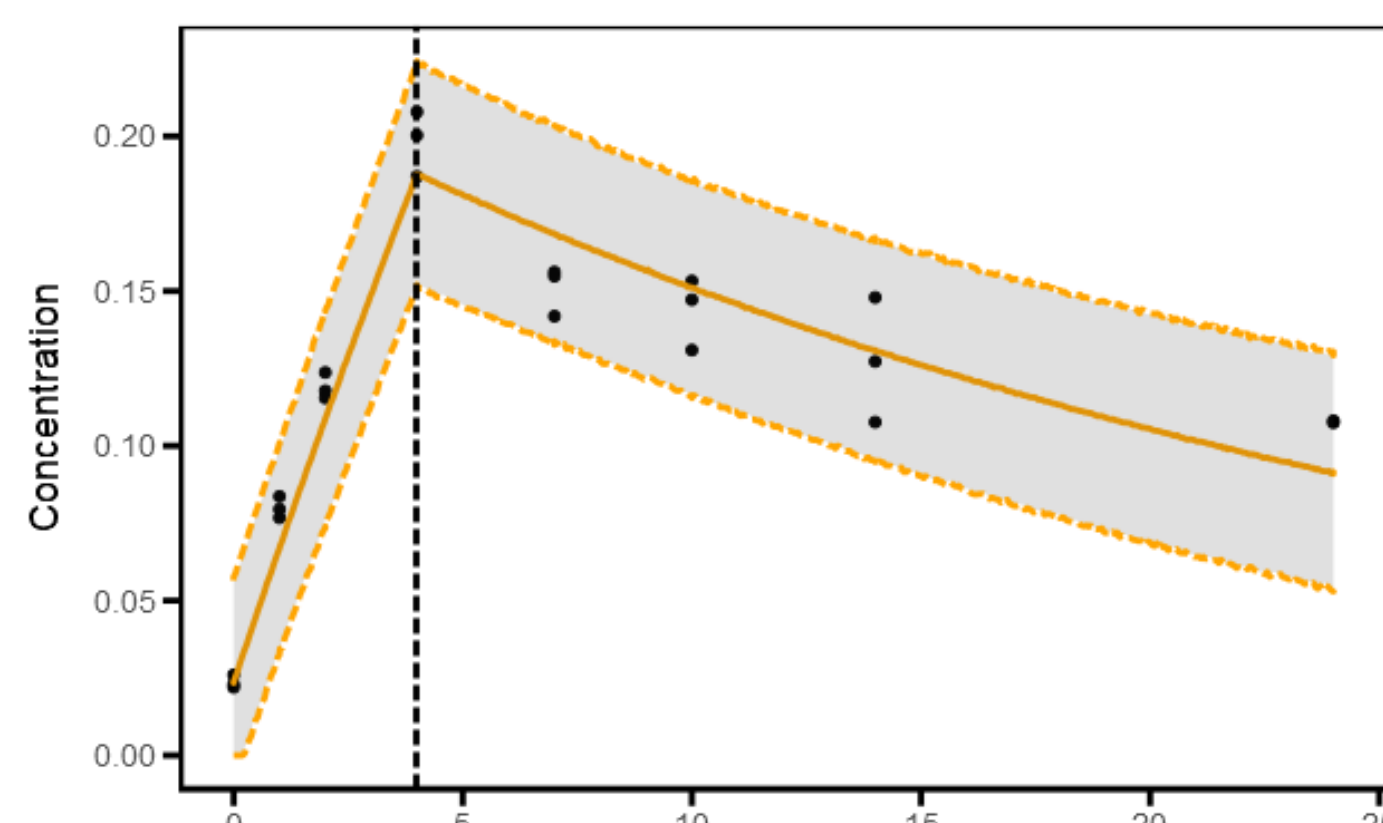

q Goodness-of-fit eriteria

Figure 4. Model predictions.
Table 2. Model parameters results. Quantiles $\$ 2.5 \% \nrightarrow 50 \% \gg 97.5 \% \gg$ Units

\section{GOODNESS-OF-FIT CRITERIA}

- The Posterior Predictive Check (PPC) let you quickly see if $95 \%$ of the oserved data are in the predicted $95 \%$ credibility intervals, as expected (Fig. 5)

- The posterior distributions (Fig. 5, in orange) indicate if your data bring enough information compared to the prior distributions (Fig. 5, in gray).

- You can also check for correlations between parameters.

- The potential scale reduction factor (PSRF) is espected to be close 1 for all parameters.

- The MCMC traces confirm the convergence of the Bayesian algorithm.
PPC

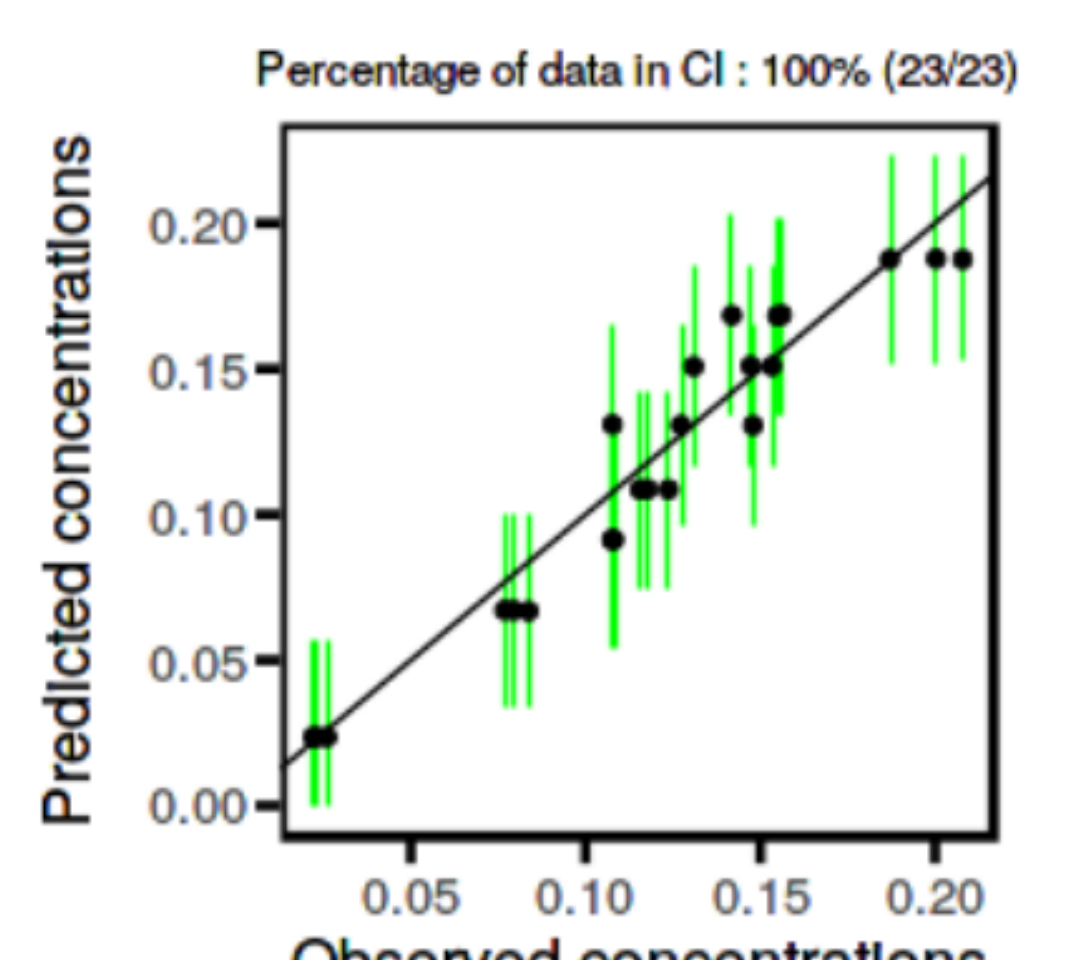

Figure 5. Goodness-of-fit criteria provided by MOSAIC ${ }_{\text {bioacc }}$
Priors and Posteriors

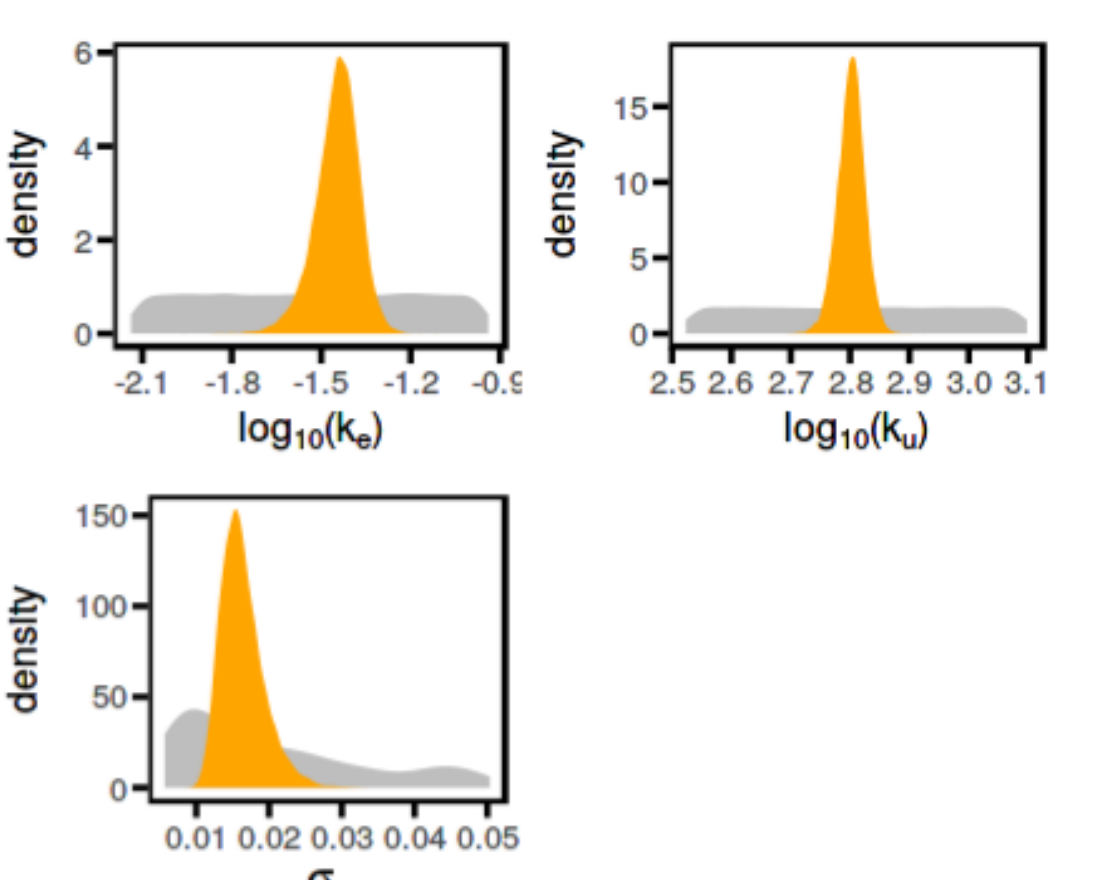

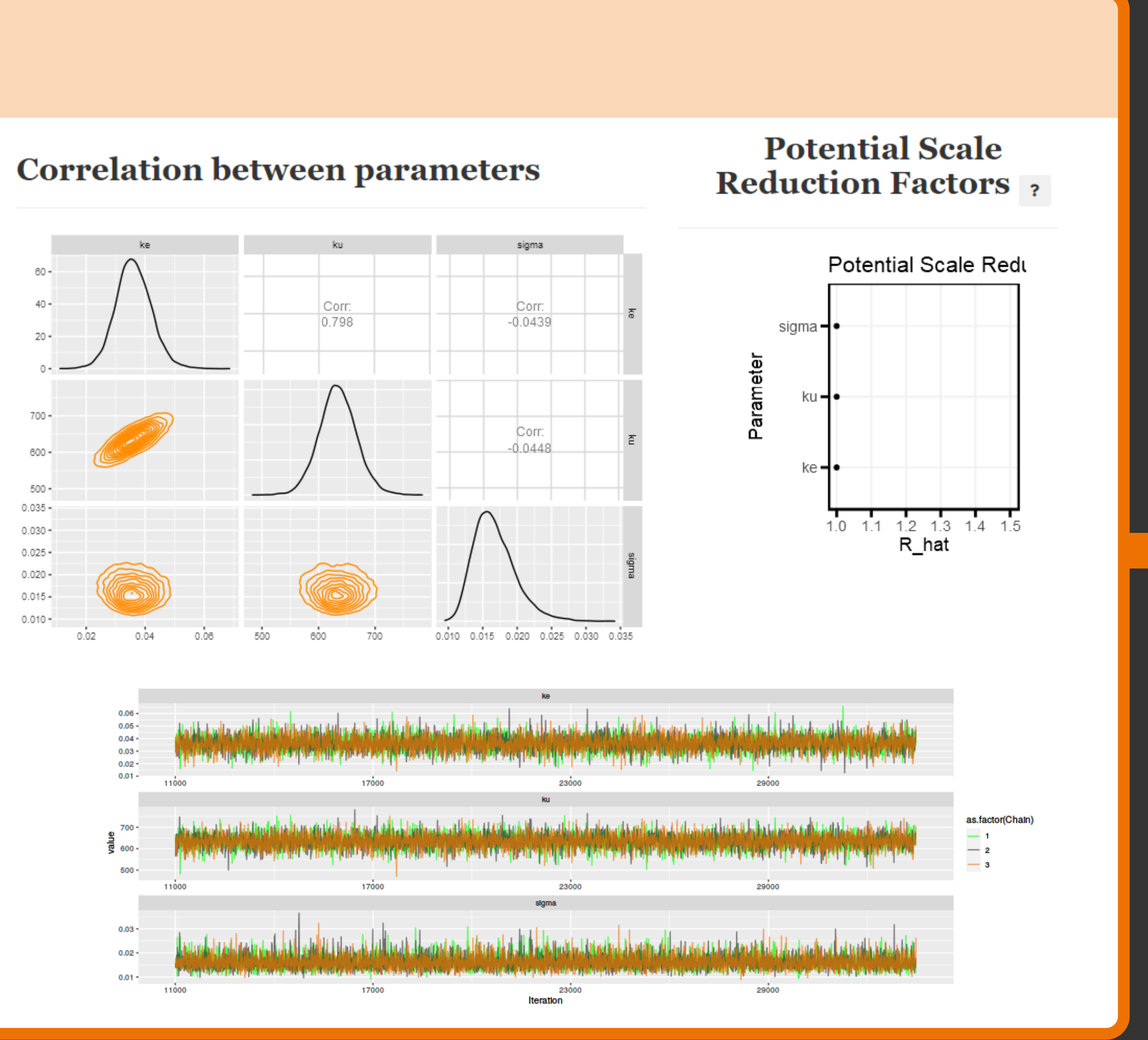

\section{References}

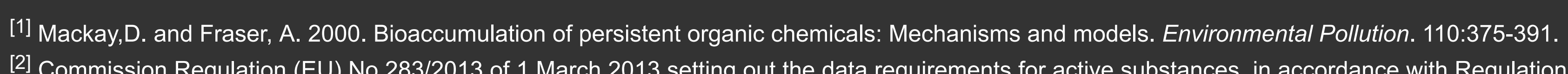

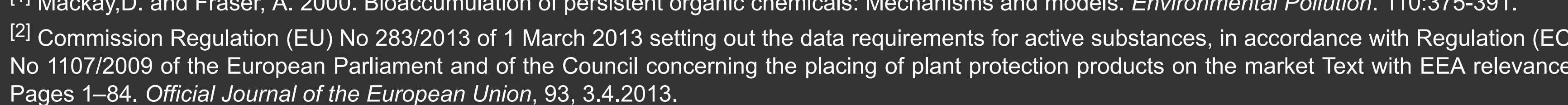
${ }^{31}$ OCDE. 2012. Test No. 305: Bioaccumulation in Fish: Aqueous and Dietary Exposure

Acknowledgements

\section{Contact Information}

We are thankful to the aquatic ecotoxicology French research group (GDR EA) for the different exchanges and to ANSES for providing the inancial support. This work was made under the umbrella of the yon" (UdL), as part of the program "Investissements d'Avenir" run by cnrs

\section{CONCLUSIONS}

- MOSAIC bioacc $_{\text {is a ready-to-use on-line }}$ tool allowing to easily perform BCF calculation, as needed by regulators for will be included in order to also calculate the BMF and the BAF, thus accounting for exposure routes and In a near future, this tool will be offered as a new module of the MOSAIC platform directly from this link:

Want to be aware? Contact us!
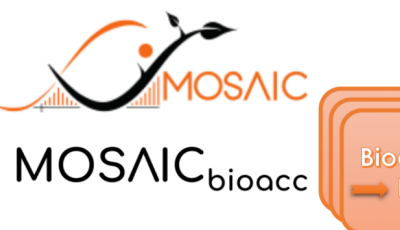